\title{
OPEN Transcriptomes in peripheral blood of young females with temporomandibular joint osteoarthritis
}

\author{
Jeong-Hyun Kang $\odot$
}

This study aimed to investigate immune-related pathophysiology of the temporomandibular joint (TMJ) osteoarthritis (OA) in young females by analyzing transcriptional profiles of peripheral blood mononuclear cells. The RNA-sequencing (RNA-seq) was conducted on 24 young females with TMJ OA (mean age $19.3 \pm 3.1$ years) (RNAOA) and 11 age and sex matched healthy controls (mean age $20.5 \pm 3.7$ years) (CON). RNA-seq datasets were analyzed to identify genes, pathways, and regulatory networks of those which were involved in the development of TMJ OA. RNA-seq data analysis revealed 41 differentially expressed genes (DEGs) between RNAOA and CON. A total of 16 gene ontology (GO) terms including three molecular and 13 biological terms were annotated via the GO function of molecular function and biological process. Through ingenuity pathway analysis (IPA), 21 annotated categories of diseases and functions were identified. There were six hub genes which showed significant results in both GO enrichment analysis and IPA, namely HLA-C, HLA-F, CXCL8, IL11RA, IL13RA1, and FCGR3B. The young females with TMJ OA showed alterations of the genes related to immune function in the blood and some of changes may reflect inflammation, autoimmunity, and abnormalT cell functions.

Osteoarthritis (OA) is characterized by degradation of components of the extracellular matrix within the articular cartilage and simultaneous remodeling of the underlying subchondral bone, in association with low inflammatory changes ${ }^{1}$. The epidemiology of OA of the temporomandibular joint (TMJ) is different from that of OA of the hand, knee, or hip joint, the incidence of which is associated with aging. Previous reports have already described female preponderance and early onset of the disease, especially from the pubertal phases to the age of early $20 \mathrm{~s}^{2,3}$. The pathophysiology of the TMJ OA is multifactorial and complex, which includes diverse etiological factors such as prolonged parafunctional habits, abnormal occlusal relationship, sustained masticatory muscle tension, and hormonal imbalance ${ }^{4,5}$. Given the early onset of the condition, TMJ OA cannot be considered to be just a simple degenerative disease such as the OA of other joints associated with the aging process, so other etiological factors could be assumed. Nevertheless, the clear pathophysiology of the TMJ OA, particularly in young patients has not yet been elucidated so far.

Many emerging evidences have demonstrated the role of immune modulation mechanisms in the development and progression of $\mathrm{OA}^{6-13}$. These processes involved immune-modulating agents, in both innate and adaptive compartments such as cytokines, chemokines, T cells, and B cells ${ }^{6-13}$. TMJ OA has been considered to be a low inflammatory arthritic condition and mainly depends on inflammation and elevated levels of inflammatory mediators and cytokines in the TMJ synovial fluid ${ }^{14-17}$. Even though one report suggested the possibility of involvement of systemic immune dysfunction in occurrence of TMJ OA ${ }^{18}$, the influences of systemic immune function and composition of immune cells in the peripheral blood on incidence and progression of TMJ OA have not been fully clarified.

The RNA sequencing (RNA-seq) technology has been utilized as a powerful tool to discover potential molecular mechanisms or therapeutic targets in various diseases ${ }^{19}$. However, to the best of the knowledge, there are yet no studies which adopted RNA-seq technology to patients with TMJ OA. Therefore, the molecular and genetic background of TMJ OA remains obscure, and the clinical treatment effects for TMJ OA are limited. Understanding comprehensive molecular profiling of TMJ OA is an essential step in discovering new candidate target molecules that are potentially involved in the pathogenesis of TMJ OA. Hence, the aim of the present study 


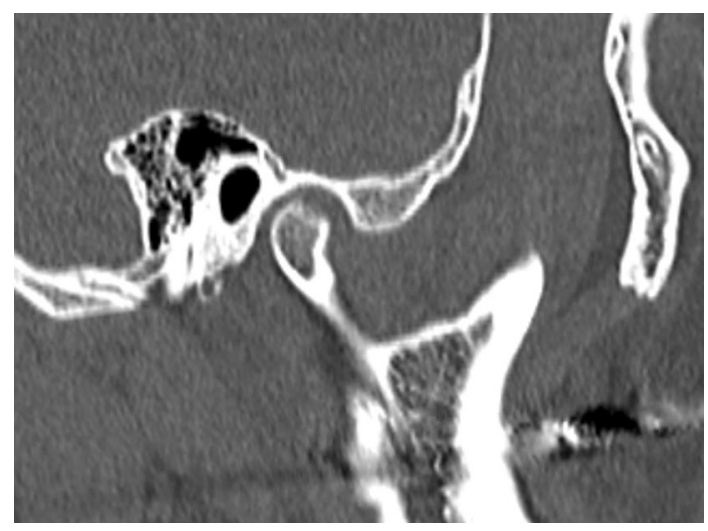

Figure 1. Sagittal cuts of CT which showed erosive osseous changes of TMJ.

was to investigate the role of the immune-related pathophysiology of TMJ OA by analyzing the transcriptional profiles of RNA from peripheral blood mononuclear cells (PBMC), which identify the differentially expressed genes (DEGs) of young females with TMJ OA.

\section{Materials and methods}

Participants. In the present study, a total of 35 young females (mean age $19.7 \pm 3.1$ years; age range 15-25 years) were enrolled. Twenty-four female patients (mean age 19.3 \pm 3.1 years; age range 15-25 years) with TMJ OA on at least one side of the condyle were consequently recruited from those who attended the TMD and Orofacial Pain Clinic at a university hospital from January, 2019 to November, 2019 (RNAOA). Eleven young females (mean age 20.5 \pm 3.7 years; age range 15-25 years) without any sign of TMD and/or TMJ OA who voluntarily participated in the study served as control (CON). Patients with the following conditions were excluded: history of head and neck trauma prior to at least 6 months prior to study entry; autoimmune diseases, which could affect the systemic levels of inflammation including juvenile idiopathic arthritis, lupus erythematosus, ankylosing spondylitis; craniofacial anomalies; and neurodegenerative disorders. All participants in the RNAOA did not show any sign of capsule or myofascial pain in the orofacial area for at least 3 months before enrollment and had not been suffered from chronic pain which last for more than 3 months in other part of the body; hence the effect of pain condition on RNA transcription profiles could be excluded. Clinical parameters such as the degree of pain free opening and maximum unassisted opening as well as the duration of TMD symptoms including TMJ noise and difficulties in opening and/or closing the mouth were evaluated. Participants were diagnosed following the Diagnostic Criteria for Temporomandibular Disorder Axis $\mathrm{I}^{20,21}$. All female participants in the RNAOA showed erosive osseous bony changes on computed tomography (CT) images indicating the area of discontinuation of the cortical lining and adjacent bone (Fig. 1). The participants who showed TMJ condyles with osteophyte or combined changes with proliferation, deformed contour, and generalized sclerosis were excluded to rule out the patients with TMJ OA after bony remodeling. No participants showed positive sites on palpation on the temporalis and masseter muscles and TMJ capsules. The research protocol was approved by the Institutional Review Board of the University Hospital (AJIRB-MED-GEN-18-449). Informed consents were obtained from all participants or, if participants were under 18, from their parents and/or legal guardians. All methods were performed in accordance with the relevant ethical guidelines and regulations.

RNA extraction from PBMC and RNA seq. To minimize the effect of circadian variation and menstruation cycle, peripheral blood was collected from all participants between 9:00 a.m. and 11:00 a.m. during their mid-luteal phases. For each individual, $3 \mathrm{ml}$ of peripheral blood was put into a PAXgene Blood RNA tube vacutainer tube (Qiagen, New York, USA). PBMC from the venous blood from all participants were isolated using Ficolle-Paque PLUS (Sigma-Aldrich). TRIzol reagent (Thermo Fisher Scientific Invitrogen Inc., MA, USA) was used to isolate the total RNA from PBMC. Genomic DNA contamination was removed using RNase-free DNase I. The amount of total RNA was measured using NanoDrop 2000 (Thermo Fisher Scientific Invitrogen Inc., MA, USA) and the integrity and quality of total RNA samples were analyzed using an Agilent Technologies 2100 Bioanalyzer (Agilent Technologies, Inc., CA, USA). All samples passed quality control RNA integrity analysis $(\mathrm{RIN} \geq 7)$. cDNA libraries were constructed using the TruSeq Stranded Total RNA Sample Preparation Kit (Illumina Inc, USA) according to the manufacturer's protocol. To produce 100 bp paired-end reads, the total RNA was sequenced using the Illumina NovaSeq 6000 system (Macrogen, Seoul, Korea). After sequencing, the indexed samples were demultiplexed before the generation of FASTQ files for analysis and assessed by FastQC version 0.11.7.

Using HISAT2 version 2.1.0 with the best score matches reported for each read, all libraries were aligned to hg19 assembly of the human genome. The mapped reads were assembled using STRING Tie version 1.3.4d. Inter-gene expression comparisons were based on calculated fragments per kilobase of transcript per million (FPKM) mapped reads. 


\begin{tabular}{|l|l|l|c|}
\hline & CON $(\mathbf{n}=1 \mathbf{1 1})$ & RNAOA $(\mathbf{n}=\mathbf{2 4})$ & $\boldsymbol{P}$ value \\
\hline Age & $23.2 \pm 11.4$ & $19.3 \pm 3.1$ & 0.268 \\
\hline BMI & $20.6 \pm 2.2$ & $20.5 \pm 2.7$ & 0.740 \\
\hline Duration of TMD symptoms (months) & 0 & $16.4 \pm 28.0$ & $<0.001^{* *}$ \\
\hline Pain free opening (mm) & $47.5 \pm 4.7$ & $42.8 \pm 5.7$ & $0.036^{*}$ \\
\hline Maximum unassisted opening $(\mathrm{mm})$ & $47.5 \pm 4.7$ & $42.8 \pm 5.7$ & $0.036^{*}$ \\
\hline
\end{tabular}

Table 1. Demographic features and TMD characteristics of participants. Descriptive values are shown as mean \pm SD. Data obtained from Mann-Whitney U tests. ${ }^{\star} P<0.05,{ }^{* \star} P<0.001$ by Mann-Whitney U tests.

Differential expression analysis of RNA-seq data. The expression level was normalized by calculating FPKM mapped reads. For DEG analysis, the value of $\log _{2}$ (fold change) were calculated. The DEGs with an adjusted $P \leq 0.05$ and $\log _{2}$ (fold changes) $\leq-1$ or $\geq 1$ were determined. Using heatmap function, the hierarchical clustering of the expression profiles of detected DEGs was conducted.

Bioinformatics analysis. Using online tool STRING analysis (http://string-db.org, version 11.0), gene ontology (GO) pathway enrichment analysis was conducted to compare gene transcription patterns between RNAOA and CON and assess the functional association between encoded proteins. False discovery rate (FDR) adjusted $P$ values were calculated for each enriched biological pathway and the threshold was set to an adjusted $P<0.01$. Gene set enrichment analysis (GSEA, version 4.0.3, http://www.gsea-msigdb.org) was used to compare gene dysregulation patterns in RNAOA with those in CON.

The threshold was set to an unadjusted $P<0.05$ to fully explore the results by ingenuity pathway analysis (IPA; Ingenuity System Inc., Redwood City, CA, USA). Using IPA, biological processes, canonical pathways, and networks of analysis were analyzed. An enrichment score measures the overlap of observed and predicted gene sets. A z-score assesses the match of observed and predicted regulation patterns, serving as a predictor of the activation state of the identified molecule.

Quantitative real time polymerase chain reaction validation. To validate the differential expression, gene expression levels were examined using quantitative real time polymerase chain reaction (qRT-PCR). qRT-PCR reactions were conducted via the Step ONE Plus (ABI, Life Technologies, CA, USA) using SYBR premix EX Taq II (Applied Biosystems, Foster City, CA, USA), and cDNA was synthesized using $1 \mu \mathrm{g}$ of mRNA. The gene expression results were obtained using the formula $2^{-(\Delta \mathrm{Ct})}$, and the fold change was calculated by the formula $2^{-(\Delta \Delta \mathrm{Ct})}$. The $\mathrm{qRT}$-PCR values were normalized using the average of the expression of the reference gene, GAPDH. Finally, the averaged fold ratios from the reference housekeeping gene were used as the relative mRNA level. Each experiment was conducted in triplicate. Two reactions, one without template and one without reverse transcriptase were also performed.

Statistical analysis. The differences in demographic features and TMD characteristics between CON and RNAOA were evaluated using Mann-Whitney U tests. The differences in gene expression patterns were defined as fold changes. Statistical analysis was achieved by setting the change in gene expression threshold to an unadjusted $P<0.01$ for encoded protein functional network analysis (FNA). The threshold was set to an unadjusted $P<0.05$ to fully explore the results by IPA. To compare gene expression levels obtained by qRT-PCR between RNA OA and CON, Mann-Whitney U tests were utilized. The level of significance was set at $P<0.05$.

\section{Results}

Demographic features and TMD symptoms in participants. The differences in age $(P=0.268)$ and body mass index $(P=0.740)$ did not show statistical significance between CON and RNAOA. Participants in RNAOA showed 16.4 \pm 28.0 months of TMD symptom duration and the amount of pain free opening and maximum unassisted opening showed a significant difference between CON and RNAOA $(P=0.036)$ (Table 1).

Gene dysregulation in peripheral blood of patients with TMJ OA. There were a total of 27,685 transcripts, and transcripts with an FPKM value less than 5 were excluded, leaving 12,788 transcripts to be analyzed. After excluding the following FPKM baseline gene, 440 genes were differentially expressed in the RNAOA compared with those in the CON ( $\mid$ fold change $\mid>1, P<0.05$ ). A total of 41 genes were expressed significantly differentially between RNAOA and CON with 17 genes upregulated and 24 downregulated in the RNAOA ( $\log 2$ (fold change) $\geq 1$ or $\leq-1, P<0.05$ ) (Table 2). A heatmap using hierarchical clustering analysis showing the expression levels of each of these genes per individual was provided (obtained from GSEA, version 4.0.3) (Fig. 2). The genes with the highest fold change in RNAOA were FGFR2, EREG, WTH3DI, CXCL8, and LINC02458 and those with the lowest fold change were SYNGR1, CYP27A1, IL11RA, MAVS, and MIR941-2 (Table 2).

Functional network analysis. The FNA of proteins encoded by differentially expressed gene transcripts $(\mathrm{n}=41, P<0.05)$ were assessed using the STRING portal (http://string-db.org, version 11.0). A total of $16 \mathrm{GO}$ terms $(P<0.01)$ including three molecular and 13 biological terms were annotated by the GO function of molecular function and biological process (Fig. 3). Four clusters of strong functional associations with significant lev- 


\begin{tabular}{|c|c|c|c|}
\hline Gene symbol & Gene name & $\log _{2}$ (fold change) & $P$ value \\
\hline FGFR2 & fibroblast growth factor receptor 2 & 1.51 & 0.020 \\
\hline EREG & epiregulin & 1.33 & 0.029 \\
\hline WTH3DI & RAB6C-like & 1.21 & 0.027 \\
\hline CXCL8 & C-X-C motif chemokine ligand 8 & 1.19 & 0.048 \\
\hline LINC02458 & long intergenic non-protein coding RNA 2458 & 1.18 & 0.041 \\
\hline SNORD133 & small nucleolar RNA, C/D box 133 & 1.13 & 0.032 \\
\hline FCER1A & Fc fragment of IgE receptor Ia & 1.13 & 0.036 \\
\hline MS4A3 & membrane spanning 4-domains A3 & 1.12 & 0.042 \\
\hline RPL21 & ribosomal protein L21 & 1.10 & 0.012 \\
\hline ITGB8 & integrin subunit beta 8 & 1.08 & 0.004 \\
\hline GPR82 & G protein-coupled receptor 82 & 1.08 & 0.049 \\
\hline LOC105377267 & uncharacterized LOC105377267 & 1.07 & 0.024 \\
\hline SNORA1 & small nucleolar RNA, H/ACA box 1 & 1.05 & 0.025 \\
\hline SNORA104 & small nucleolar RNA, H/ACA box 104 & 1.05 & 0.020 \\
\hline MS4A2 & membrane spanning 4-domains A2 & 1.04 & 0.017 \\
\hline HRH4 & histamine receptor $\mathrm{H} 4$ & 1.03 & 0.029 \\
\hline ACAT2 & acetyl-CoA acetyltransferase 2 & 1.03 & 0.039 \\
\hline GAS5-AS1 & GAS5 antisense RNA 1 & 1.02 & 0.032 \\
\hline DOCK1 & dedicator of cytokinesis 1 & 1.01 & 0.020 \\
\hline CA8 & carbonic anhydrase 8 & 1.01 & 0.020 \\
\hline IRAK1BP1 & interleukin 1 receptor associated kinase 1 binding protein 1 & 1.01 & 0.002 \\
\hline CPA3 & carboxypeptidase A3 & 1.01 & 0.018 \\
\hline GTSF1 & gametocyte specific factor 1 & 1.01 & 0.049 \\
\hline ZNF257 & zinc finger protein 257 & 1.00 & 0.031 \\
\hline HLA-F & major histocompatibility complex, class I, F & -1.00 & 0.014 \\
\hline HSPA6 & heat shock protein family A (Hsp70) member 6 & -1.01 & 0.003 \\
\hline NCF1C & neutrophil cytosolic factor 1C pseudogene & -1.01 & 0.047 \\
\hline HLA-C & major histocompatibility complex, class I, C & -1.02 & 0.022 \\
\hline IL13RA1 & interleukin 13 receptor subunit alpha 1 & -1.02 & 0.034 \\
\hline TNFRSF8 & TNF receptor superfamily member 8 & -1.02 & 0.026 \\
\hline FCGR3B & Fc fragment of IgG receptor IIIb & -1.03 & 0.050 \\
\hline TMEM176B & transmembrane protein $176 \mathrm{~B}$ & -1.03 & 0.047 \\
\hline MIR941-3 & microRNA 941-3 & -1.03 & 0.019 \\
\hline TNFRSF25 & TNF receptor superfamily member 25 & -1.03 & 0.039 \\
\hline CCHCR1 & coiled-coil alpha-helical rod protein 1 & -1.04 & 0.042 \\
\hline UTS2 & urotensin 2 & -1.04 & 0.043 \\
\hline SYNGR1 & synaptogyrin 1 & -1.06 & 0.030 \\
\hline CYP27A1 & cytochrome P450 family 27 subfamily A member 1 & -1.11 & 0.007 \\
\hline IL11RA & interleukin 11 receptor subunit alpha & -1.11 & 0.044 \\
\hline MAVS & mitochondrial antiviral signaling protein & -1.14 & 0.029 \\
\hline MIR941-2 & microRNA 941-2 & -1.46 & 0.031 \\
\hline
\end{tabular}

Table 2. Transcripts with altered gene expression in the RNAOA compared to CON.

els of network enrichment ( $P=0.000821)$ were observed: (1) HLA-C, HLA-F, TNFRSF25, CCHCR1, FCER1A, MS4A2, and CPA3, (2) FGFR2, EREG, CXCL8, and HRH4, and UTS2 (3) FCGR3B and TNFRSF8, and (4) IL11RA and IL13RA1 (Fig. 4).

Pathway analysis using IPA. IPA was carried out on the transcriptome dataset with significance set at $P<0.05$ and $\mid \log 2$ (fold change) $\mid>1$. IPA identified 20 annotated categories of diseases and functions that were significantly upregulated ( $\mathrm{z}$-score $>2.0$ ) and included 13 of the DEGs from the study. The 5 top canonical pathways were associated with the role of natural killer cells, communication between innate and adaptive immune cells, and T cell signaling (Table 3). Nine genes were involved in five top canonical pathways namely, FCGR3B, HLA-C, HLA-F, HSPA-6, CXCL8, ITGB8, FCER1A, IL11RA, and IL13RA1. Hence, these pathways reinforce significant immune and inflammatory dysregulation occurring in the pathogenesis of TMJ OA. 


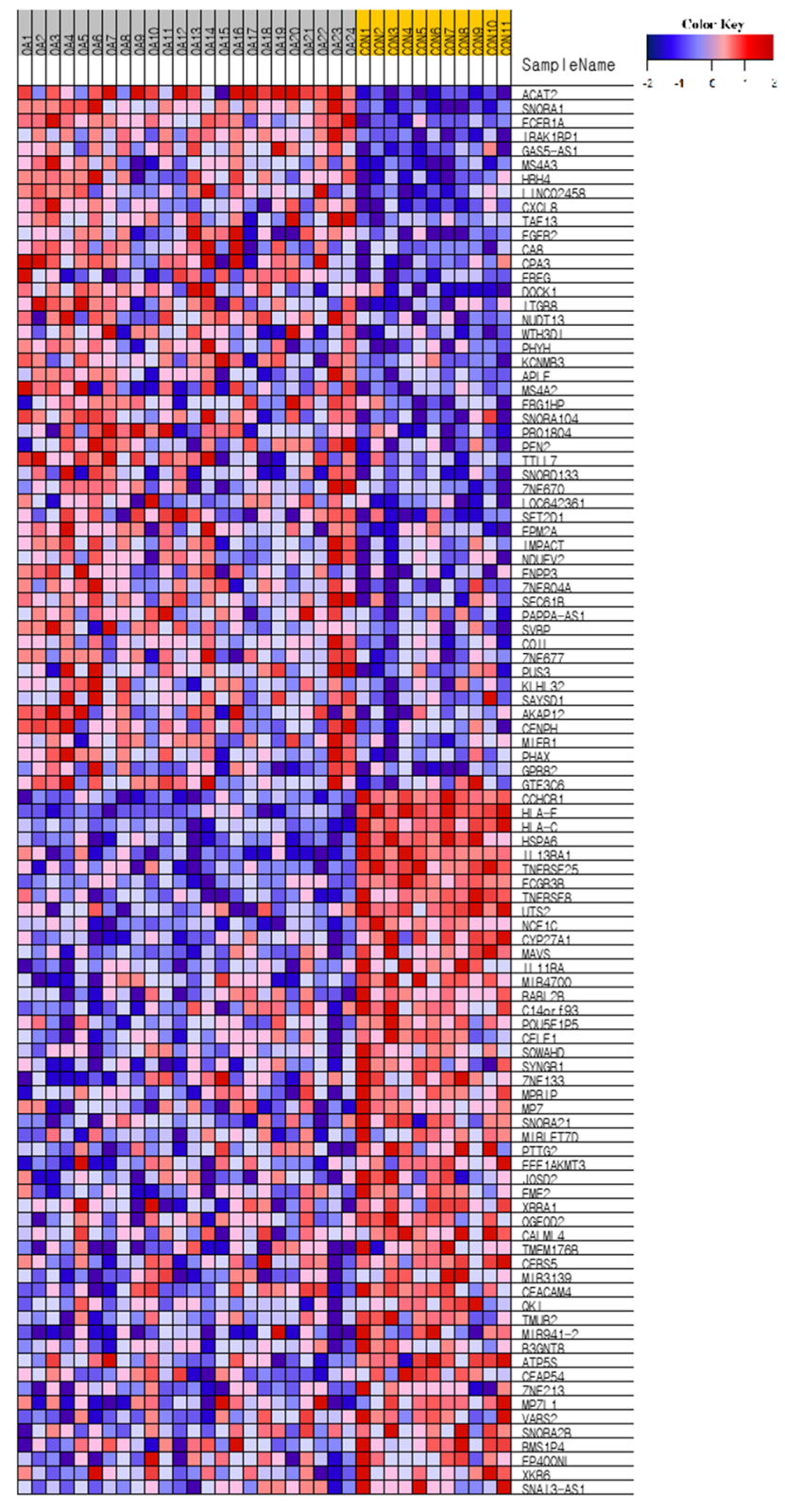

Figure. 2. Heatmap demonstrating the expression patterns of DEGs between RNAOA and CON. The color key at the top indicates relative gene expression. Red and blue colors represent higher and lower gene expression levels, respectively. The figure was obtained from GSEA, version 4.0.3 (http://www.gsea-msigdb.org).

qRT-PCR. The gene expression patterns of 6 hub genes, including HLA-C, HLA-F, CXCL8, IL11RA, IL13RA1, and FCGR3B transcripts which showed significant results in both GO enrichment analysis and IPA were tested via qRT-PCR. Significant differences were observed in HLA-C $(P=0.030)$, CXCL8 $(P=0.022)$, and IL11RA transcripts $(P=0.018)$ (Fig. 5).

\section{Discussion}

Early onset of diseases, especially during pubertal phases and age of early $20 \mathrm{~s}$ is a unique feature of the TMJ $\mathrm{OA}^{2,3}$. The progression of the TMJ OA may accompany compromised masticatory function and altered craniofacial morphology, which could affect an individual's quality of life ${ }^{22-24}$. However, to date, the exact pathophysiology of TMJ OA particularly in young patients and clear molecular mechanisms of the development of TMJ OA have not yet been elucidated, so far. Immune dysfunction has been considered as one of the main etiological factors which may result in bony destruction in patients with OA from other joints ${ }^{6-9,12,13}$, but studies which clearly elucidate the immunological background of TMJ OA especially in young patients are sparse. RNA-seq is an effective tool for clarifying molecular mechanisms in genetic levels and could provide the novel therapeutic targets involved in a certain condition ${ }^{19}$. To the best of the knowledge, no study ever attempted to reveal the immune related etiology of TMJ OA using RNA-seq technology. Consequently, the aim of the present study was 


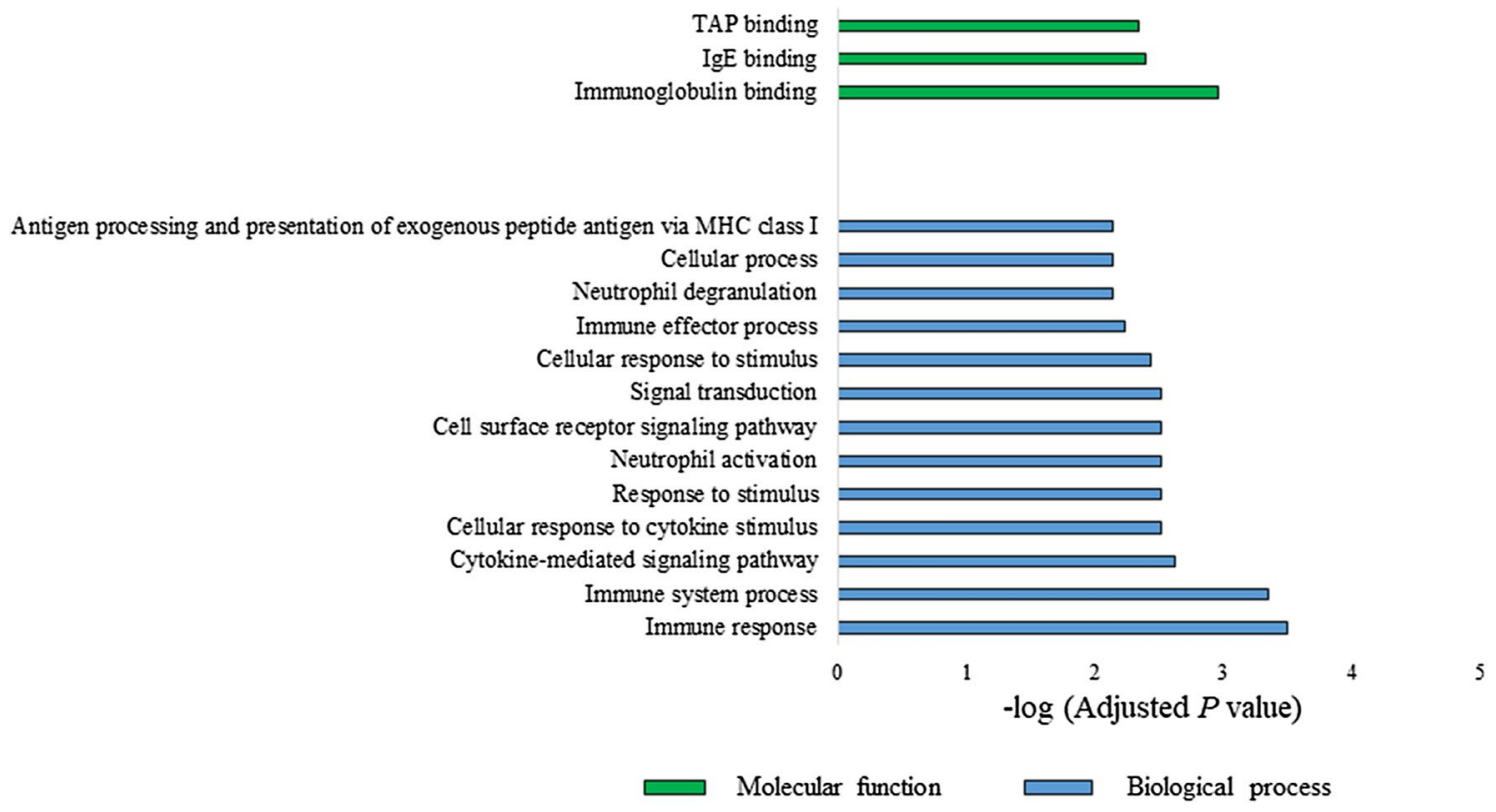

Figure 3. GO pathway enrichment analysis of dysregulated genes in peripheral blood of young females with TMJ OA. A total of $16 \mathrm{GO}$ terms $(P<0.01)$ including 3 molecular and 13 biological terms were annotated using the GO function of molecular function and biological process.

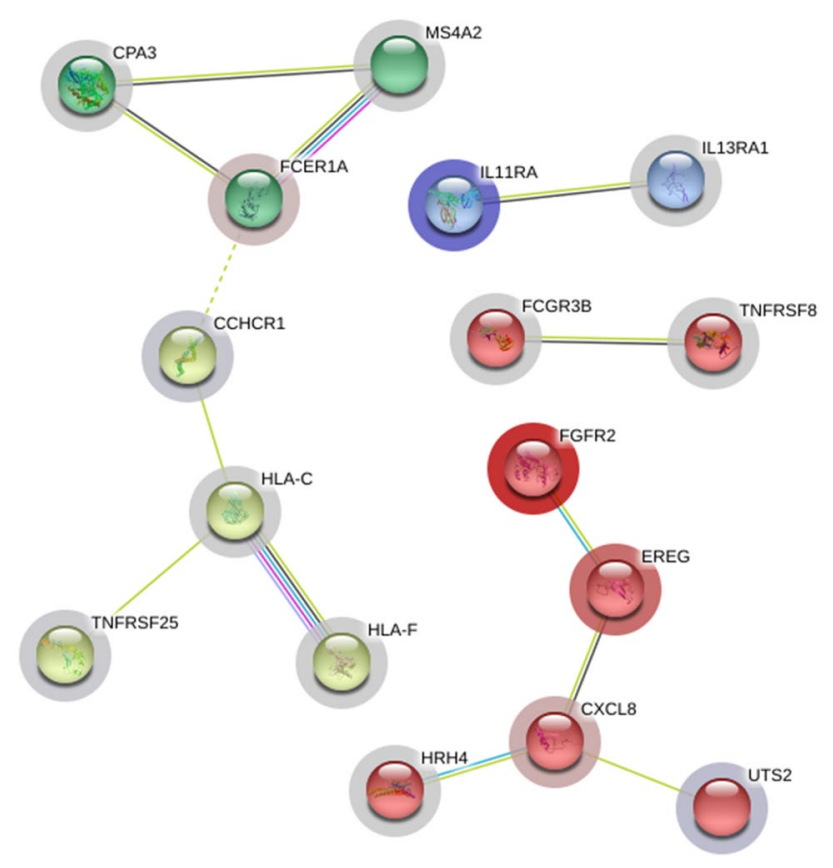

Figure 4. Four clusters of strong functional associations with significant levels of network enrichment. The figure was obtained from STRING portal (http://string-db.org, version 11.0).

to investigate the role of immune dysfunction by analyzing transcriptional profiles of PBMC and search for new therapeutic targets for management of TMJ OA in young patients.

Declined acquired immune responses accompanied by increased autoreactivity have been detected in the elderly ${ }^{25}$ and relationships between this altered innate immune function, $\mathrm{T}$ cell and $\mathrm{B}$ cell responses, and cartilage and bone degradation have been reported ${ }^{26,27}$. Aforementioned results from IPA demonstrated associations among various autoimmune disorders, including autoimmune thyroid disease, systemic lupus erythematosus (SLE), and occurrence of TMJ OA. Previous studies already have mentioned the high prevalence of temporomandibular disorders in patients with autoimmune thyroid disorders or SLE ${ }^{28-30}$. Even though severe condylar resorption in patients with rheumatoid arthritis or juvenile idiopathic arthritis has been detected ${ }^{31}$, few reports ever mentioned the bony changes of condyles in other autoimmune diseases such as autoimmune thyroid disorders 


\begin{tabular}{|l|l|l|}
\hline Ingenuity canonical pathways & Z-score & Molecules \\
\hline Natural killer cell signaling & 3.63 & FCGR3B, HLA-C, HLA-F, HSPA6 \\
\hline Communication between innate and adaptive immune cells & 3.36 & CXCL8, HLA-C, HLA-F \\
\hline Virus entry via endocytic pathways & 3.23 & HLA-C, HLA-F, ITGB8 \\
\hline White adipose tissue browning pathway & 2.99 & FCER1A, FGFR2, MS4A2 \\
\hline STAT3 pathway & 2.93 & FGFR2, IL11RA, IL13RA1 \\
\hline Cytotoxic T lymphocyte-mediated apoptosis of target cells & 2.9 & HLA-C, HLA-F \\
\hline Antigen presentation pathway & 2.78 & HLA-C, HLA-F \\
\hline Tec kinase signaling & 2.69 & FCER1A, MS4A2, TNFRSF25 \\
\hline Graft-versus-Host disease signaling & 2.61 & HLA-C, HLA-F \\
\hline Autoimmune thyroid disease signaling & 2.59 & HLA-C, HLA-F \\
\hline Role of NFAT in regulation of the immune response & 2.57 & FCER1A, FCGR3B, MS4A2 \\
\hline Dendritic cell maturation & 2.56 & FCGR3B, HLA-C, HLA-F \\
\hline Systemic lupus erythematosus signaling & 2.28 & FCGR3B, HLA-C, HLA-F \\
\hline Cardiac hypertrophy signaling (enhanced) & 2.19 & CXCL8, FGFR2, IL11RA, IL13RA1 \\
\hline Allograft rejection signaling & 2.11 & HLA-C, HLA-F \\
\hline CTLA4 signaling in cytotoxic T lymphocytes & 2.08 & HLA-C, HLA-F \\
\hline Crosstalk between dendritic cells and natural killer cells & 2.08 & HLA-C, HLA-F \\
\hline Protein ubiquitination pathway & 2.07 & HLA-C, HLA-F, HSPA6 \\
\hline OX40 signaling pathway & 2.07 & HLA-C, HLA-F \\
\hline Fcy receptor-mediated phagocytosis in macrophages and monocytes & 2.04 & DOCK1, FCGR3B \\
\hline
\end{tabular}

Table 3. Annotated categories of diseases and functions from IPA.

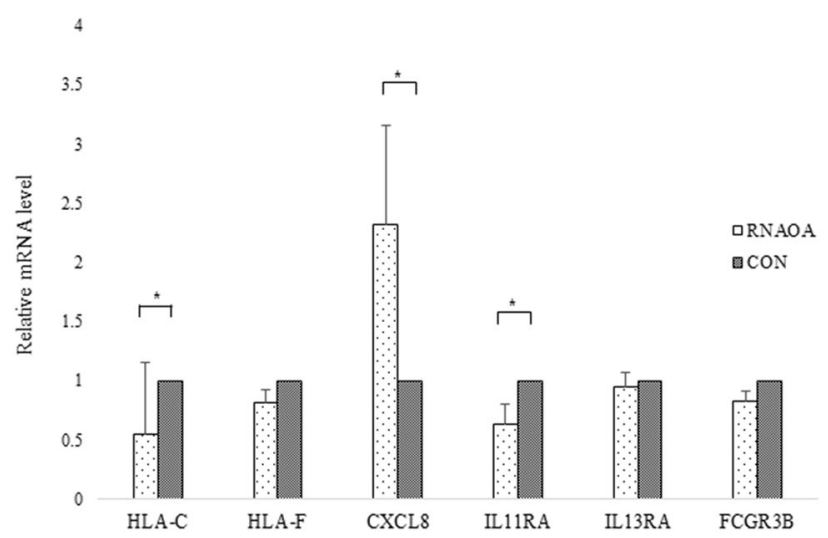

Figure 5. Validation of RNA-seq results using qRT-PCR. Normalized fold expressions of mRNA are shown. Error bar represents mean \pm standard error. Mann-Whitney $U$ tests were used to compare gene expression levels $\left({ }^{\star} P<0.05\right)$.

or SLE. Because patients with autoimmune disorders were excluded from the present study, clear associations between autoimmune thyroid disorders or SLE and TMJ OA could not be clarified. Even though, previous studies which dealt with the associations between innate immunity and OA progression focused on increased autoreactivity related to the aging process, the role of altered innate immune function in bony destruction of TMJ condyles even in young females could be assumed through this study.

Accumulating evidence suggested the involvement of inflammatory and immune responses in the pathogenesis of $\mathrm{OA}^{32}$. The results from the present study demonstrated the increased expression levels of chemokine (C-X-C motif) ligand 8 (CXCL8), the involvement of signal transducers and activators of transcription 3 (STAT3) pathway, and cytokine mediated immune response in the development of TMJ OA in young females. Interleukin-8/ CXCL8 (IL-8/CXCL8) has been found to be an attractant for neutrophils and a population of lymphocytes ${ }^{33}$. The increased level of IL-8 in synovial fluid from the knee OA and TMD patients with disc displacement has been reported ${ }^{34-37}$, but the mechanisms of IL-8 in subchondral bone changes in TMJ have not been clearly revealed. One study has reported elevated serum levels of CXCL8 and expression levels of STAT3 in knee OA patients ${ }^{38}$. This study suggested that CXCL8 may inactivate the mitosis of chondrocytes and further aggravate OA progression and indirectly induce the Janus kinase (JAK)/STAT3 signaling in chondrocytes. Although the specific molecular mechanisms of CXCL8 and STAT3 could not be revealed in the present results, the associations 
between CXCL8, STAT3 signaling, and subchondral bony destruction of TMJ condyles in young females could be suspected.

The peripheral blood of patients with OA have been analyzed and revealed that patients with OA have shown altered levels of CD8 $+\mathrm{T}$ cells and more cytotoxic profiles in comparison with healthy controls ${ }^{11,39-41}$. One animal study suggested the molecular mechanisms of the role of CD8 + T cells in the OA process that CD8 + T cells were activated once OA had been initiated and cartilage degeneration occurred more slowly in CD8 + T cell knockout mice than in wild-type ${ }^{42}$. The results from this study also showed the relationships between the occurrence of TMJ OA and T cell activity through increased expression levels of genes related to Tec kinase signaling, nuclear factor of activated T cells (NFAT) regulation, and OX40 signaling. Furthermore, cytotoxic T lymphocyte mediated apoptosis and CTLA4 signaling pathway which have an association with cytotoxic $\mathrm{T}$ cell function, seem to have roles in the incidence of TMJ OA. A previous study has focused on the role of T helper cell in synovial fluid in the subchondral bony changes in TMJ OA patients ${ }^{16}$, but no study ever attempted to clarify altered cytotoxic $\mathrm{T}$ cell activities in blood in patients with TMJ OA.

Due to cross-sectional study design, the causal relationships between altered RNA transcriptional profiles and development of TMJ OA could not be derived from this study. The altered transcriptional profile would be result from TMJ bony destruction, and also abnormal immune-modulation function could be one of the main etiological factors of the TMJ OA. Because TMJs are small peripheral joints, the bony changes of the TMJs may not induce prominent changes in the systemic transcriptional profiles in the peripheral blood. However, regarding the statistically significant differences in the selected pathway and detected genes and sample number over 30 , those differences could not be results just from chance.

To the best of our knowledge, the present study is the first study which attempted to reveal the genetic and molecular background of TMJ OA in young patients using the RNA-seq technology. Several previous reports which dealt with the systemic immune dysfunction and the development of OA have focused on the role of the aging process and the senescence of immune cells. In addition, most of the studies which analyzed the relationships between levels of the immune-modulating agents in blood and OA dealt with weight bearing axial joints such as the knee and hip not with peripheral joints. Aforementioned results showed that altered systemic immune function could have a role in the development of arthritic conditions even in young patients with OA from small peripheral joints such as TMJ. However, the present study still has several limitations, first of all, RNA-seq was conducted using only PBMC, not using the synovial tissue. However, because TMJ is small joint, synovial fluid collecting process which accompanies with puncturing process inevitably causes iatrogenic inflammation in the small joint space and this may affect the RNA transcriptional profiles. Furthermore, the aim of this study was to figure out the immune-related pathophysiology of TMJ OA in young females, so adopting peripheral blood would be essential to reveal the purpose of this study. Secondly, only female participants were included. Even though previous studies confirmed the female preponderance of TMJ OA, the absence of male participants would limit the understanding of the genetic etiology of the TMJ OA. Thirdly, owing to the cross-sectional characteristics of the study, the information regarding the changes in the transcriptome profiles in accordance with the progression or recovery of TMJ OA could not be provided. Finally, the lack of control with inflammatory arthritis including juvenile idiopathic arthritis and rheumatoid arthritis could give limited information. However, no study which tried to reveal the immune-related pathophysiology of the TMJ OA based on transcriptional profiles of the peripheral blood have been investigated. Future prospective RNA-seq studies with large samples including both male and female participants and another control with inflammatory arthritis would be necessary.

In conclusion, in the present study, young female TMJ OA patients showed alterations in the blood immune cell expression and some of the changes may reflect inflammation, autoreactivity, and altered $\mathrm{T}$ cell functions. For proper management and successful treatment of TMJ OA in young females, future research regarding immunemodulation based therapy would be warranted.

\section{Data availability}

The datasets used and/or analyzed during the current study are available from the corresponding author on reasonable request.

Received: 3 January 2021; Accepted: 9 April 2021

Published online: 23 April 2021

\section{References}

1. Zarb, G. A. \& Carlsson, G. E. Temporomandibular disorders: Osteoarthritis. J. Orofac. Pain 13, 295-306 (1999).

2. Kim, K., Wojczynska, A. \& Lee, J. Y. The incidence of osteoarthritic change on computed tomography of Korean temporomandibular disorder patients diagnosed by RDC/TMD; a retrospective study. Acta Odontol. Scand. 74, 337-342. https://doi.org/10. 3109/00016357.2015.1136678 (2016).

3. Manfredini, D., Piccotti, F., Ferronato, G. \& Guarda-Nardini, L. Age peaks of different RDC/TMD diagnoses in a patient population. J. Dent. 38, 392-399. https://doi.org/10.1016/j.jdent.2010.01.006 (2010).

4. Tanaka, E., Detamore, M. S. \& Mercuri, L. G. Degenerative disorders of the temporomandibular joint: Etiology, diagnosis, and treatment. J. Dent. Res. 87, 296-307. https://doi.org/10.1177/154405910808700406 (2008).

5. Wang, X. D., Zhang, J. N., Gan, Y. H. \& Zhou, Y. H. Current understanding of pathogenesis and treatment of TMJ osteoarthritis. J. Dent. Res. 94, 666-673. https://doi.org/10.1177/0022034515574770 (2015).

6. Haseeb, A. \& Haqqi, T. M. Immunopathogenesis of osteoarthritis. Clin. Immunol. 146, 185-196. https://doi.org/10.1016/j.clim. 2012.12.011 (2013).

7. Kapoor, M., Martel-Pelletier, J., Lajeunesse, D., Pelletier, J. P. \& Fahmi, H. Role of proinflammatory cytokines in the pathophysiology of osteoarthritis. Nat. Rev. Rheumatol. 7, 33-42. https://doi.org/10.1038/nrrheum.2010.196 (2011).

8. Li, Y. S., Luo, W., Zhu, S. A. \& Lei, G. H. T cells in osteoarthritis: Alterations and beyond. Front. Immunol. 8, 356. https://doi.org/ 10.3389/fimmu.2017.00356 (2017). 
9. Lopes, E. B. P., Filiberti, A., Husain, S. A. \& Humphrey, M. B. Immune contributions to osteoarthritis. Curr. Osteoporos. Rep. 15, 593-600. https://doi.org/10.1007/s11914-017-0411-y (2017).

10. Lurati, A., Laria, A., Gatti, A., Brando, B. \& Scarpellini, M. Different T cells' distribution and activation degree of Th17 CD4+ cells in peripheral blood in patients with osteoarthritis, rheumatoid arthritis, and healthy donors: Preliminary results of the MAGENTA CLICAO study. Open Access Rheumatol. 7, 63-68. https://doi.org/10.2147/OARRR.S81905 (2015).

11. Ponchel, F. et al. Changes in peripheral blood immune cell composition in osteoarthritis. Osteoarthritis Cartilage 23, 1870-1878. https://doi.org/10.1016/j.joca.2015.06.018 (2015).

12. Weber, A., Chan, P. M. B. \& Wen, C. Do immune cells lead the way in subchondral bone disturbance in osteoarthritis?. Prog. Biophys. Mol. Biol. 148, 21-31. https://doi.org/10.1016/j.pbiomolbio.2017.12.004 (2019).

13. Woodell-May, J. E. \& Sommerfeld, S. D. Role of inflammation and the immune system in the progression of osteoarthritis. J. Orthop. Res. 38, 253-257. https://doi.org/10.1002/jor.24457 (2020).

14. Kacena, M. A. et al. Inflammation and bony changes at the temporomandibular joint. Cells Tissues Organs 169, 257-264. https:// doi.org/10.1159/000047889 (2001).

15. Kellesarian, S. V. et al. Cytokine profile in the synovial fluid of patients with temporomandibular joint disorders: A systematic review. Cytokine 77, 98-106. https://doi.org/10.1016/j.cyto.2015.11.005 (2016).

16. Monasterio, G. et al. Th1/Th17/Th22 immune response and their association with joint pain, imagenological bone loss, RANKL expression and osteoclast activity in temporomandibular joint osteoarthritis: A preliminary report. J. Oral. Rehabil. 45, 589-597. https://doi.org/10.1111/joor.12649 (2018).

17. Xiong, H. et al. Elevated leptin levels in temporomandibular joint osteoarthritis promote proinflammatory cytokine IL-6 expression in synovial fibroblasts. J. Oral Pathol. Med. 48, 251-259. https://doi.org/10.1111/jop.12819 (2019).

18. Nishioka, M. et al. TMJ osteoarthritis/osteoarthrosis and immune system factors in a Japanese sample. Angle Orthod. 78, 793-798. https://doi.org/10.2319/091407-438 (2008).

19. Wang, Z., Gerstein, M. \& Snyder, M. RNA-Seq: A revolutionary tool for transcriptomics. Nat. Rev. Genet. 10, 57-63. https://doi. org/10.1038/nrg2484 (2009).

20. Ahmad, M. et al. Research diagnostic criteria for temporomandibular disorders (RDC/TMD): Development of image analysis criteria and examiner reliability for image analysis. Oral Surg. Oral Med. Oral Pathol. Oral Radiol. Endod. 107, 844-860. https:// doi.org/10.1016/j.tripleo.2009.02.023 (2009).

21. Schiffman, E. et al. Diagnostic criteria for temporomandibular disorders (DC/TMD) for clinical and research applications: Recommendations of the international RDC/TMD consortium network* and orofacial pain special interest Groupdagger. J. Oral Fac. Pain Headache 28, 6-27. https://doi.org/10.11607/jop.1151 (2014).

22. Bertram, S., Moriggl, A., Neunteufel, N., Rudisch, A. \& Emshoff, R. Lateral cephalometric analysis of mandibular morphology: Discrimination among subjects with and without temporomandibular joint disk displacement and osteoarthrosis. J. Oral Rehabil. 39, 93-99. https://doi.org/10.1111/j.1365-2842.2011.02251.x (2012).

23. Bertram, S., Moriggl, A., Rudisch, A. \& Emshoff, R. Structural characteristics of bilateral temporomandibular joint disc displacement without reduction and osteoarthrosis are important determinants of horizontal mandibular and vertical ramus deficiency: A magnetic resonance imaging study. J. Oral Maxillofac. Surg. 69, 1898-1904. https://doi.org/10.1016/j.joms.2010.12.026 (2011).

24. Kang, J. H., Yang, I. H., Hyun, H. K. \& Lee, J. Y. Dental and skeletal maturation in female adolescents with temporomandibular joint osteoarthritis. J. Oral Rehabil. 44, 879-888. https://doi.org/10.1111/joor.12547 (2017).

25. Tomer, Y. \& Shoenfeld, Y. Ageing and autoantibodies. Autoimmunity 1, 141-149. https://doi.org/10.3109/08916938809001927 (1988).

26. Garnero, P. et al. Cross sectional evaluation of biochemical markers of bone, cartilage, and synovial tissue metabolism in patients with knee osteoarthritis: Relations with disease activity and joint damage. Ann. Rheum Dis. 60, 619-626. https://doi.org/10.1136/ ard.60.6.619 (2001).

27. Pazar, B. et al. Basic calcium phosphate crystals induce monocyte/macrophage IL-1beta secretion through the NLRP3 inflammasome in vitro. J. Immunol. 186, 2495-2502. https://doi.org/10.4049/jimmunol.1001284 (2011).

28. Grozdinska, A., Hofmann, E., Schmid, M. \& Hirschfelder, U. Prevalence of temporomandibular disorders in patients with Hashimoto thyroiditis. J. Orofac. Orthop. 79, 277-288. https://doi.org/10.1007/s00056-018-0140-6 (2018).

29. Song, H. S. et al. Association between temporomandibular disorders, chronic diseases, and ophthalmologic and otolaryngologic disorders in Korean adults: A cross-sectional study. PLoS ONE 13, e0191336. https://doi.org/10.1371/journal.pone.0191336 (2018).

30. Aliko, A., Ciancaglini, R., Alushi, A., Tafaj, A. \& Ruci, D. Temporomandibular joint involvement in rheumatoid arthritis, systemic lupus erythematosus and systemic sclerosis. Int. J. Oral Maxillofac. Surg. 40, 704-709. https://doi.org/10.1016/j.ijom.2011.02.026 (2011).

31. Dolwick, F. M. Arthritis and allied conditions-a textbook of rheumatology 2019-2025 (Lippincott Williams \& Wilkins, 2001).

32. Creamer, P. \& Hochberg, M. C. Osteoarthritis. Lancet 350, 503-508. https://doi.org/10.1016/S0140-6736(97)07226-7 (1997).

33. Baggiolini, M., Walz, A. \& Kunkel, S. L. Neutrophil-activating peptide-1/interleukin 8, a novel cytokine that activates neutrophils. J. Clin. Invest. 84, 1045-1049. https://doi.org/10.1172/JCI114265 (1989).

34. Koh, S. M. et al. Elevated plasma and synovial fluid interleukin-8 and interleukin-18 may be associated with the pathogenesis of knee osteoarthritis. Knee 27, 26-35. https://doi.org/10.1016/j.knee.2019.10.028 (2020).

35. Pierzchala, A. W., Kusz, D. J. \& Hajduk, G. CXCL8 and CCL5 expression in synovial fluid and blood serum in patients with osteoarthritis of the knee. Arch. Immunol. Ther. Exp. (Warsz) 59, 151-155. https://doi.org/10.1007/s00005-011-0115-4 (2011).

36. Matsumoto, K. et al. Cytokine profile in synovial fluid from patients with internal derangement of the temporomandibular joint: a preliminary study. Dentomaxillofac. Radiol. 35, 432-441. https://doi.org/10.1259/dmfr/77288976 (2006).

37. Sato, J. et al. Expression of interleukin 8 in synovial tissues in patients with internal derangement of the temporomandibular joint and its relationship with clinical variables. Oral Surg. Oral Med. Oral Pathol. Oral Radiol. Endod. 103, 467-474. https://doi.org/ 10.1016/j.tripleo.2006.06.058 (2007).

38. Yang, P. et al. Expression profile of cytokines and chemokines in osteoarthritis patients: Proinflammatory roles for CXCL8 and CXCL11 to chondrocytes. Int. Immunopharmacol. 40, 16-23. https://doi.org/10.1016/j.intimp.2016.08.005 (2016).

39. Kuryliszyn-Moskal, A. Comparison of blood and synovial fluid lymphocyte subsets in rheumatoid arthritis and osteoarthritis. Clin. Rheumatol. 14, 43-50. https://doi.org/10.1007/BF02208083 (1995).

40. Apinun, J. et al. Immune mediators in osteoarthritis: Infrapatellar fat pad-infiltrating CD8+ T cells are increased in osteoarthritic patients with higher clinical radiographic grading. Int. J. Rheumatol. 2016, 9525724. https://doi.org/10.1155/2016/9525724 (2016).

41. Sae-Jung, T. et al. Functional and T cell receptor repertoire analyses of peripheral blood and infrapatellar fat pad $\mathrm{T}$ cells in knee osteoarthritis. J. Rheumatol. 46, 309-317. https://doi.org/10.3899/jrheum.170775 (2019).

42. Hsieh, J. L. et al. CD8+ T cell-induced expression of tissue inhibitor of metalloproteinses-1 exacerbated osteoarthritis. Int. J. Mol. Sci. 14, 19951-19970. https://doi.org/10.3390/ijms141019951 (2013).

\section{Acknowledgements}

This study was supported by the National Research Foundation of Korea (NRF) grant funded by the Korea government (2018R1C1B6007671). Figure 2 was obtained from obtained from GSEA, version 4.0.3. Figure 4 
was obtained from STRING portal (http://string-db.org, version 11.0). Figure 1, 3, and 5 were prepared by the single author (JHK).

\section{Author contributions}

A single author J.-H.K. contributed to the obtaining of funding, conception and design of the article, collection of data, analysis of data, interpretation of data, construction of manuscript, and prepared the figures.

\section{Competing interests}

The author declares no competing interests.

\section{Additional information}

Correspondence and requests for materials should be addressed to J.-H.K.

Reprints and permissions information is available at www.nature.com/reprints.

Publisher's note Springer Nature remains neutral with regard to jurisdictional claims in published maps and institutional affiliations.

(c) (i) Open Access This article is licensed under a Creative Commons Attribution 4.0 International License, which permits use, sharing, adaptation, distribution and reproduction in any medium or format, as long as you give appropriate credit to the original author(s) and the source, provide a link to the Creative Commons licence, and indicate if changes were made. The images or other third party material in this article are included in the article's Creative Commons licence, unless indicated otherwise in a credit line to the material. If material is not included in the article's Creative Commons licence and your intended use is not permitted by statutory regulation or exceeds the permitted use, you will need to obtain permission directly from the copyright holder. To view a copy of this licence, visit http://creativecommons.org/licenses/by/4.0/.

(C) The Author(s) 2021 